
Cahiers
de a Recherche
Four es Droits
Fondamentaux
Cahiers de la recherche sur les droits fondamentaux

16 | 2018

Les partis politiques

\title{
Vers un nouveau modèle partisan ? Entre déclassement des partis de gouvernement et avènement des partis-mouvements
}

Is There a Move Towards a New Partisan Model? Between a Displacement of Government Parties and the Appearance of Parties-Movements?

\section{Rémi Lefebvre}

\section{OpenEdition}

Journals

Édition électronique

URL : https://journals.openedition.org/crdf/301

DOI : $10.4000 /$ crdf.301

ISSN : 2264-1246

Éditeur

Presses universitaires de Caen

Édition imprimée

Date de publication : 16 novembre 2018

Pagination : 21-30

ISBN : 978-2-84133-901-3

ISSN : $1634-8842$

\section{Référence électronique}

Rémi Lefebvre, «Vers un nouveau modèle partisan ? Entre déclassement des partis de gouvernement et avènement des partis-mouvements ", Cahiers de la recherche sur les droits fondamentaux [En ligne], 16 | 2018, mis en ligne le 16 novembre 2019, consulté le 14 novembre 2022. URL : http:// journals.openedition.org/crdf/301; DOI : https://doi.org/10.4000/crdf.301 


\title{
Vers un nouveau modèle partisan? Entre déclassement des partis de gouvernement et avènement des partis-mouvements
}

\author{
Rémi LEFEBVRE \\ Professeur de science politique à l'université de Lille \\ Centre d'études et de recherches administratives, politiques et sociales (CERAPS, UMR 8026)
}

I. À quoi servent (encore) les partis traditionnels?
A. Le modèle dominant du parti électoral professionnel
B. Les primaires ouvertes: levier de re-légitimation ou de destruction des partis traditionnels?

II. Les partis-mouvements: un modèle stabilisé?
A. Le parti-plateforme
B. Des cultures militantes opposées

Les élections présidentielles et législatives de 2017 ont décomposé puis recomposé le système partisan. Cette transformation sera-t-elle durable ${ }^{1}$ ? Elle est en tout cas profonde. Jusque janvier 2017, une alternance électorale sans surprise semblait programmée. Sur la base des sondages, l'élection du candidat LR (Les Républicains), François Fillon, désigné dans le cadre d'une primaire ayant mobilisé près de 4 millions d'électeurs, semblait inéluctable. L'élection a déjoué, on le sait, ce scénario. Un novice en politique, jamais passé sous les fourches caudines du suffrage universel et inconnu quelques mois avant l'élection, a accédé à la fonction suprême. Quelques semaines plus tard, il peut s'appuyer sur une large majorité parlementaire puisque, son mouvement, La République en marche, remporte contre toute attente largement les élections législatives (308 députés). Les candidats des deux partis de gouvernement qui exerçaient le pouvoir dans une alternance quasi automatique depuis $1981^{2}$ et semblaient verrouiller le jeu politique n'ont pas réussi à se qualifier pour le second tour de l'élection présidentielle. Les deux formations ont rallié à peine un quart des votants au premier tour. Le Parti socialiste (PS) a connu une débâcle historique, Benoît Hamon ne rassemblant que $6,5 \%$ aux élections présidentielles et le groupe parlementaire socialiste passant de 295 à 31 députés. Cette victoire d'Emmanuel Macron et d'En marche procède de logiques conjoncturelles et contingentes (le «Penelope Gate» ou affaire Fillon, la défection de François Hollande...) mais aussi de mécanismes plus structurels. Emmanuel Macron n'a pas à lui seul bouleversé le système partisan. La centralité des partis de gouvernement tenait beaucoup aux règles du jeu institutionnel et au mode de scrutin majoritaire (le parti dominant raflant tout avec la captation croissante du scrutin législatif par l'élection présidentielle). La victoire du ministre de l'Économie de

1. L'électorat d'Emmanuel Macron est un précipité hétérogène qui a agrégé à la faveur de l'élection des sous-électorats divers.

2. Le système politique a connu l'alternance en 1981, 1986, 1988, 1993, 1997, 2002 et 2012. Une forme de cohabitation local-national s'est mise en place, le parti dans l'opposition remportant systématiquement les élections intermédiaires. 
François Hollande ne marque pas seulement la déroute du PS et de LR mais consacre aussi la faillite d'un modèle organisationnel: le «parti politique traditionnel»3. L'élection présidentielle a agi ainsi comme un «accélérateur de transition ${ }^{4}$, le système partisan marquant déjà des signes de crise. Elle n'a pas donné lieu à une crise institutionnelle (Emmanuel Macron s'est au contraire pleinement appuyé sur les ressources du régime) mais a révélé et accentué une crise (ancienne) du système des partis ${ }^{5}$ qui a favorisé l'irruption partidaire ${ }^{6} \mathrm{~d}^{\prime} \mathrm{En}$ marche.

Les partis politiques constituent les institutions politiques qui suscitent la défiance la plus forte des français. Selon les baromètres du Centre de recherches politiques de Sciences Po (CEVIPOF), 85 \% des Français ne leur font pas confiance. La perception dominante des organisations partisanes est qu'elles servent essentiellement les ambitions de leurs dirigeants, que les «appareils» partisans, repliés sur leurs jeux et enjeux propres, sont dominés quasi exclusivement par des enjeux de pouvoir et qu'ils cultivent des oppositions stériles ou artificielles. Le discrédit sans précédent des professionnels de la politique rejaillit sur les partis qui sont un des rouages d'une démocratie représentative et d'un système électoral à la légitimité de plus en plus contestée $^{7}$. Cette critique des partis politiques n'est pas nouvelle ${ }^{8}$ mais elle s'est en quelque sorte radicalisée. Elle est désormais portée par les partis politiques eux-mêmes qui depuis des années cherchent à se « rénover», se «refonder», se « régénérer ${ }^{9} \ldots$

La situation des partis politiques est de fait paradoxale. Leur place dans le système politique s'est à la fois effritée et renforcée. Leurs périmètres d'action se sont rétrécis. De moins en moins ancrés dans la société et assis sur des bases militantes peu représentatives, les partis ne sont plus des médiateurs d'intérêts sociaux autour desquels s'organise le débat public. Ils ne constituent plus, notamment à gauche, des intellectuels collectifs structurant l'opinion publique et administrant un sens politique. Pourtant, leur position dans le jeu politique et surtout électoral demeure centrale. Ils constituent le principal opérateur de professionnalisation politique. L'investiture partisane constitue, à tous les niveaux institutionnels ${ }^{10}$, un sésame indispensable pour entrer en politique et solliciter avec des chances de succès les suffrages des électeurs. Le financement public des partis politiques a conforté cette place institutionnelle des partis politiques devenus des «agences semi-publiques» de la démocratie électorale ${ }^{11}$.

L'émergence de partis se présentant comme des mouvements ou des entreprises comme La République en marche ou La France insoumise est de ce point de vue particulièrement éclairante. Ces organisations se sont construites contre les partis traditionnels et proposent des modèles en rupture avec les pratiques traditionnelles mais n'en conservent pas moins certains traits des partis politiques. Prenant juridiquement la forme de parti, elles ont été créées par en haut pour asseoir les ambitions présidentielles de leur leader autoproclamé et ont investi des candidats aux élections législatives selon des procédures centralisées inédites. La victoire d'Emmanuel Macron n'a à ce titre pas dérogé à une des «lois» de la $\mathrm{V}^{\mathrm{e}}$ République qui postule qu'un candidat crédible doit s'appuyer sur un parti ou une machine électorale. La politique demeure donc une affaire de partis mais reste à savoir desquels? Entre affaiblissement des partis traditionnels et émergence des mouvements-entreprises plateformes, on montrera ici que la forme partisane semble se réinventer sans qu'un modèle précis ne se stabilise encore (on ne prétend pas ici forger un nouveau modèle analytique de parti).

\section{I. À quoi servent (encore) les partis traditionnels?}

Les partis politiques sont nés à la fin du XIX $\mathrm{X}^{\mathrm{e}}$ siècle avec l'émergence de la civilisation électorale. «Enfants du suffrage universel», selon l'expression classique de Max Weber, ils concourent à l' «expression électorale» (Constitution de 1958) et structurent le jeu politique tant au niveau territorial (la mobilisation et la politisation des électeurs) qu'au niveau parlementaire (ils contribuent à coordonner voire à discipliner l'activité des élus). Les labels partisans permettent aux électeurs de se repérer dans l'offre politique. De manière plus spécifique, le parti, entendu comme entreprise idéologique et militante, est, à gauche de l'espace politique, une invention du mouvement ouvrier. Il constitue, comme l'écrit Roberto Michels, «l'arme dont disposent les faibles dans la lutte contre les forts $»^{12}$. Dans la « démocratie du public», qui caractérise le gouvernement représentatif contemporain ${ }^{13}$, les sphères

3. Cette catégorie n'a guère de sens. Elle subsume des réalités politiques très hétérogènes et ne vaut que par opposition à des nouvelles organisations jugées plus «souples» ou se donnant à voir comme «modernes».

4. P. Martin, «La présidentielle 2017, accélérateur de transition», The Conversation, 10 mai 2017.

5. Voir P. Raynaud, Emmanuel Macron: une révolution bien tempérée, Paris, Desclée de Brouwer, 2018.

6. Emmanuel Macron évoque « une forme de brutalité de l'histoire, une effraction» (Libération, 20 février 2018)

7. Pierre Rosanvallon évoque dans ses travaux récents «le déclin de la performance démocratique des élections» ou de leur fonction représentative pointant par là leur incapacité croissante à produire la légitimité des gouvernants (voir Le bon gouvernement, Paris, Seuil, 2015).

8. Depuis leur création à la fin du XIX ${ }^{\mathrm{e}}$ siècle, les partis sont accusés de "diviser», de produire des dérives idéologiques, oligarchiques...

9. Savoir/agir, $\mathrm{n}^{\mathrm{O}}$ 32, juin 2015, Réinventer les partis politiques, N. Éthuin, R. Lefebvre (dir.).

10. La part des élus non membres d'un parti recule depuis les années 1970 y compris au niveau municipal.

11. Pour reprendre les catégories du modèle du «parti cartel» dominant dans la littérature anglo-saxonne.

12. R. Michels, Sociologie du parti dans la démocratie moderne: enquête sur les tendances oligarchiques de la vie des groupes [1910], traduction et présentation par J.-C. Angaut, Paris, Gallimard (Folio), 2015.

13. B. Manin, Principes du gouvernement représentatif, Paris, Flammarion, 1996, partie VI.3. Selon Bernard Manin, une désintermédiation s'opère, les médias et les sondages se substituant à la médiation partisane. 
d'action des partis se sont érodées. Les primaires ouvertes ont constitué une des stratégies de re-légitimation du PS et de la droite républicaine (Union pour un mouvement populaire - UMP -, puis LR) mais elles n'ont pas produit les effets attendus.

\section{A. Le modèle dominant du parti électoral professionnel}

Trois «fonctions» sont traditionnellement prêtées aux partis: l'intégration sociopolitique, la production idéologique et programmatique, la sélection des élites et des candidats. Cette approche fonctionnaliste a été fortement critiquée pour son systémisme ${ }^{14}$ simpliste mais s'avère utile pour analyser l'évidement des partis politiques ${ }^{15}$. La première fonction renvoie aux rapports entre le parti et la société. Les partis sont le produit de clivages sociaux et de conflits politiques (conflits de classe, public-privé, productivisme-écologie, centralisation $v s$ identités régionales...) même s'ils n'en sont pas le strict reflet. Ils sont censés porter des intérêts sociaux, les mettre en forme et en offre politique sur le marché électoral, représenter des groupes et produire des identités sociales. S'ils parviennent toujours à représenter la majorité des membres de certaines fractions de la société (l'électorat de droite est de plus en plus homogène socialement), cette dimension s'est affaiblie pour tout un ensemble de raisons. La société s'est déconflictualisée et les classes sociales ont perdu de leur consistance, subjective notamment, alors même que les inégalités sociales se sont exacerbées ${ }^{16}$. Les partis cherchent à maximiser leurs performances électorales en s'adressant à un public socialement large et interclassiste (modèle du parti attrape-tout). Les électorats partisans sont des agrégats plus fragiles (affaiblissement de la loyauté électorale à un parti et montée de la volatilité électorale). L'ancrage des partis dans la société s'est de fait affaibli. Le déclin du militantisme (plus net en France que dans le reste des pays européens) en est à la fois une cause et une conséquence. Le militantisme partisan, plus que jamais perçu comme un «embrigadement», apparaît peu attractif dans un contexte où l'offre d'engagements s'est restructurée autour de formes de participation plus ponctuelles, pragmatiques et moins hiérarchiques (au risque de la fragilité et de l'inefficacité ${ }^{17}$ ). Mais les partis politiques cherchent-ils vraiment à recruter à l'heure où la cotisation des adhérents n'est plus une source essentielle de financement et alors que la croyance que les médias font l'élection est dominante? Le modèle des "parties without partisans" est discuté dans la littérature sur les organisations partisanes ${ }^{18}$. L'engagement apparaît moins distancié (individualiste, labile...) que mis à distance par les partis. Les réseaux des partis politiques à gauche, viviers de recrutement militant, se sont du reste rétractés (réseaux laïques pour le $\mathrm{PS}^{19}$, ouvriers pour le Parti communiste - PC). L'articulation est devenue problématique entre mondes partisan, syndical, associatif, culturel ou intellectuel qui tendent chacun à se replier sur eux-mêmes dans une logique auto-référentielle, ce qui affecte la capacité de rayonnement et de politisation des partis de gauche. Ces évolutions compromettent leurs capacités d'encadrement des catégories populaires qui était une de leurs vocations historiques. Les partis tendent ainsi à flotter dans une forme d'apesanteur sociale. Le recours croissant aux sondages et aux services d'agence de communication cherche à compenser ce déficit de réceptivité et de représentativité sociale.

Les partis de gouvernement peinent à remplir la deuxième fonction: ils ne sont plus porteurs de visions de la société discriminantes et les grands récits idéologiques dans lesquels s'enchâssaient les partis ont perdu de leur force. Une forme d'indifférenciation idéologique s'est opérée dans les démocraties occidentales entre partis libéraux de droite et partis réformistes de gauche. La social-démocratie, en crise partout en Europe, s'est alignée idéologiquement sur le référentiel néolibéral et des politiques de compétitivité perçues comme des adaptations inéluctables pour faire à la mondialisation et à la course à la compétitivité. Justifiant la politique économique de François Hollande, le porte-parole du gouvernement Stéphane Le Foll déclare: "La politique de l'offre n'est ni de droite ni de gauche, elle est nécessaire ${ }^{20}$, faisant écho à Tony Blair qui, devant les députés français en 1998, assénait dans une perspective proche: «Il n'y a pas des politiques économiques de gauche ou de droite mais des politiques qui sont efficaces ou ne le sont pas». Philippe Raynaud parle à juste titre d'un "centrisme implicite» qui transcende les alternances pardelà les rhétoriques de campagne depuis les années 1980 en

14. La relation partisane fait l'objet de multiples « usages» sociaux.

15. Le risque aussi de ce modèle est d'idéaliser un âge d'or des partis qui n'a jamais existé (la France n'a jamais connu de parti de masses sauf le Parti communiste français - PCF). D’un certain point de vue, les partis «ne sont plus ce qu'ils n'ont jamais été»; voir R. Lefebvre, «Le militantisme socialiste n'est plus ce qu'il n'a jamais été. Modèle de "l'engagement distancié" et transformations du militantisme au Parti socialiste», Politix, $\mathrm{n}^{\circ} 102,2013$, p. $7-33$.

16. Les partis sont bien au cœur du paradoxe contemporain des classes sociales qu'ils contribuent eux-mêmes à défaire subjectivement. Selon Olivier Schwartz la société actuelle est à la fois moins et plus dominée par les classes sociales que par le passé; O. Schwartz, "Vivons-nous encore dans une société de classes? Trois remarques sur la société française contemporaine», La vie des idées, 22 septembre 2009.

17. On pense ici au mouvement Nuits debout qui s'est construit en partie contre les logiques de dépossession de la parole des militants produites par les partis mais qui n'est pas parvenu à durer, rejetant toute forme d'institutionnalisation.

18. Parties Without Partisans: Political Change in Advanced Industrial Democracies, R. J. Dalton, M. P. Wattenberg (dir.), Oxford - New York, Oxford University Press, 2000.

19. Voir R. Lefebvre, F. Sawicki, La société des socialistes: le PS aujourd'hui, Bellecombe-en-Bauges, Éditions du Croquant, 2006.

20. Les échos, 17 janvier 2014. 
France $^{21}$. Cette nouvelle configuration partisane a ouvert de nouveaux espaces à des partis qualifiés de manière peu rigoureuse de "populistes» ou à des organisations plus radicales à gauche (l'offre du premier tour à l'élection présidentielle de 2017 atteste d'une forte polarisation idéologique).

De manière plus générale, les partis de gauche ont désinvesti la réflexion doctrinale et le travail idéologique et tendent à externaliser ce travail à des think tanks ou des cercles d'experts. Les partis ne sont guère plus des lieux d'auto-formation. Les débats politiques et idéologiques se sont déplacés vers les arènes médiatiques, les espaces intellectuels ou les réseaux sociaux. Quand le parti exerce le pouvoir, il n'a plus de réelle autonomie idéologique par rapport aux équipes gouvernementales et ne devient plus qu'un rouage de la communication de l'exécutif. Rafaël $\operatorname{Cos}^{22} \mathrm{a}$ analysé dans une thèse de science politique récente le désinvestissement tendanciel au PS du travail partisan d'élaboration programmatique. Il montre que le travail intellectuel ou d'expertise conduit au sein du parti est globalement peu valorisé. Ceux qui l'endossent se voient accorder à la fois peu de ressources, et peu d'audience. Il n'y a plus de «travail doctrinal au long cours» au PS, et les rares moments de cristallisation idéologique mobilisent peu les dirigeants du parti, davantage préoccupés par les autres tâches liées au calendrier électoral. Il démontre aussi que les activités programmatiques en tant que telles sont beaucoup moins centrales que par le passé dans les jeux partisans: les procédures collectives d'élaboration des projets socialistes sont assez largement désinvesties; les projets constituent davantage « un simple instrument à la disposition du Premier secrétaire pour chercher à exister", soit face à l'exécutif quand il est socialiste, soit dans le cadre de la compétition pour la désignation du candidat. Il montre enfin que les programmes prennent moins sens que par le passé au sein de l'espace de la gauche. On peut lire dans sa thèse:

Ce qui borne l'écriture des textes et leur utilisation lors des campagnes électorales, c'est d'abord le poids exercé par un milieu d'exégètes professionnels composé de journalistes, de sondeurs, de thinks tanks et de certains groupes d'intérêts. Le glissement de la mise en livre à la mise en chiffres des programmes signale le poids déterminant désormais exercé par cette contrainte de crédibilité.

Il ne reste en somme aux partis que leur fonction de sélection des élites et des candidats, elle-même amoindrie. L'activité des partis politiques est de plus en plus orientée vers la maximisation de leurs résultats électoraux (la mise en discipline, non sans difficultés, des groupes ${ }^{23}$ ). Le fait n'est pas nouveau bien sûr: les partis politiques sont depuis leur émergence des groupements en concurrence sur le marché des postes. La nouveauté est sans doute qu'ils constituent désormais des entreprises largement financées sur fonds publics et que leur rationalité électorale prend largement le pas sur d'autres logiques (intégration sociale, sociabilité, construction idéologique, politisation de la société). Les partis réunissent désormais surtout des agents directement "intéressés» à l'obtention de profits électoraux ou professionnels (la place croissante des collaborateurs d'élus et de l'auxiliariat politique en témoigne). Angelo Panebianco analyse ainsi l'avènement du «parti électoral professionnel ${ }^{24}$. Le PS et LR sont devenus des machines électorales, peuplées essentiellement de professionnels de la politique, de gestionnaires locaux et d'aspirants à l'élection. Les écologistes, adeptes de la "politique autrement», sont devenus une «firme» (pour reprendre l'expression de Noël Mamère). Le fonctionnement de ces partis est principalement structuré par l'élection présidentielle qui mobilise et consume toutes les énergies. La «lutte des places» s'est intensifiée, même si le phénomène n'est pas nouveau, et les partis éprouvent des difficultés croissantes à réguler et à arbitrer ces luttes de pouvoir. Les primaires apparaissent comme un nouveau mode de production du leadership présidentiel.

\section{B. Les primaires ouvertes: levier de re-légitimation ou de destruction des partis traditionnels?}

Les primaires ouvertes, adoptées successivement par le PS (2009) et l'UMP (2013), apparaissent comme une réponse aux nouveaux défis et à la crise de légitimité auxquels les partis sont confrontés. Si les adhérents perdent la prérogative exclusive de la désignation, la procédure est bien une «affaire de partis». Ce sont les organisations partisanes qui décident en effet du déclenchement de cette consultation "privée», délimitent son périmètre, codifient les règles $\mathrm{du}$ jeu et qualifient les candidats qui peuvent y prendre part. Les primaires constituent en ce sens une tentative pour conforter les partis de gouvernement et « sécuriser» leur place dans le système politique (l'argument visant à assurer la qualification au second tour pour l'UMP face au Front national - FN - a été central dans le choix de cette procédure). Les primaires sont devenues en d'autres termes la nouvelle méthode pour s'arroger le monopole légitime des ressources afférentes à la marque partisane (le financement notamment).

21. P. Raynaud, Emmanuel Macron: une révolution bien tempérée. Emmanuel Macron a fait éclater une "synthèse» socialiste qui apparaissait de plus en plus artificielle (de Manuel Valls à Gérard Filoche pour aller vite). Il a cherché à démontrer que les clivages essentiels ne sont plus entre la gauche et la droite mais traversent les partis eux-mêmes (d'où, selon lui, des «guerres civiles » permanentes). Notons néanmoins que le PS est longtemps parvenu à faire coexister des lignes politiques très divergentes (dans les années 1970 notamment).

22. R. Cos, Les socialistes croient-ils à leurs programmes? Démobilisations et recompositions du travail programmatique au Parti socialiste (1995-2012), thèse de doctorat en science politique, université de Lille, 2017.

23. Voir (In)disciplines partisanes, A. Allal, N. Bué (dir.), Villeneuve-d'Ascq, Presses universitaires du Septentrion, 2016.

24. A. Panebianco, Political Parties: Organization and Power, Cambridge, Cambridge University Press, 1988. 
Comment expliquer l'adoption des primaires ouvertes $^{25}$ ? Elles s'inscrivent d'abord dans un processus de «démocratisation ${ }^{26}$ des partis qui les conduit dans un premier temps à donner plus de pouvoir aux militants avant d'élargir le droit de désignation aux sympathisants dans un second temps. Le préalable de la primaire ouverte c'est la primaire fermée (1995 pour le PS, 2007 pour l'UMP). Pourtant, le passage de l'une à l'autre n'a rien de naturel ni linéaire. La procédure «directe» de désignation du candidat par les militants leur confère un nouveau pouvoir et ouvre une rétribution symbolique de nature à redynamiser le militantisme. La primaire ouverte conserve le caractère direct de la sélection mais conduit à un phénomène inverse : elle retire un pouvoir au militant et tend ce faisant à démonétiser son statut. Mais, dans un contexte de montée d'un «impératif participatif», les partis cherchent, à travers l'innovation démocratique des primaires, à projeter une image d'ouverture et de modernité qui tranche avec le caractère autocentré et «stérile» des luttes internes. Ce nouveau mode de désignation est bien le produit de l'affaiblissement des partis politiques. Le rétrécissement de leur base militante, le déclin de leur ancrage et partant de leur représentativité sociale conduisent à délégitimer les modes de sélection traditionnelle. De manière générale, à mesure que se fragilise l'identification partisane, les liens entre électeurs et partis se fragilisent. Aussi les primaires constituent-elles une manière de resserrer ces liens en intégrant les électeurs dans le choix du candidat pour maximiser les chances de victoire. Il s'agit dans cette perspective d'optimiser le choix du candidat, d'accroître la légitimité des partis et leur crédibilité dans l'opinion publique, d'enrichir le choix des électeurs jusque-là limité ou de pré-mobiliser les électeurs. Une dernière évolution structurelle explique le recours aux primaires: l'hyperprésidentialisation de la vie politique, renforcée par le quinquennat et l'inversion du calendrier. Dès lors que la victoire à l'élection présidentielle devient l'ultima ratio des partis et l'alpha et l'oméga de la vie politique, structurant les stratégies d'un nombre croissant d'acteurs, les partis cherchent à aligner leur fonctionnement sur le système institutionnel.

Ces facteurs généraux doivent néanmoins être contextualisés. Dans la conversion aux primaires apparaissent déterminants des éléments conjoncturels et contingents. Les contextes dans lesquels le PS et l'UMP ont été amenés à adopter les primaires présentent des homologies structurales fortes. Dans les deux cas, la défaite (la troisième consécutive en 2008 pour le PS, celle de 2012 pour l'UMP) a produit une vacance de leadership qui exacerbe la concurrence pour les positions dirigeantes. Or les instruments traditionnels d'arbitrage ne parviennent pas à clarifier cette confrontation et un leadership incontesté fait défaut. Les fraudes lors du congrès de Reims en 2008 et lors du duel Copé / Fillon en 2012 ont disqualifié les procédures internes. Au-delà du récit irénique de la «démocratisation", les primaires s'imposent ainsi avant tout comme un mode de régulation des luttes internes et la solution procédurale permettant de les trancher dans un cadre codifié, négocié et transparent. Dans la «conversion» de la droite, les effets d'entraînement de la primaire socialiste de 2011 ont joué un rôle incontestable (effet de contagion) tout comme la montée du FN qui contraint à l'unité au premier tour pour se qualifier au deuxième.

La campagne présidentielle de 2017 a retourné l'image des primaires: elles apparaissent comme un processus périlleux voire destructeur qui n'est en aucune manière une garantie de victoire. Célébrées comme une avancée démocratique majeur et un outil de sélection performant du candidat à l'élection présidentielle (la «martingale» de la victoire de François Hollande en 2012), les primaires sont désormais plutôt considérées comme des "machines à perdre " aux effets incontrôlables et comme un facteur d'hystérisation du débat public. Dans un entretien à l'hebdomadaire Le point ${ }^{27}$, quelques semaines avant l'élection où il a renoncé à se présenter (dans une large mesure à cause des primaires), le président de la République déclarait: «Il ne doit plus $\mathrm{y}$ avoir de primaires dans des partis de gouvernement, sinon il n'y aura plus de parti de gouvernement dans ce pays». À droite comme à gauche, les primaires ont contribué à la désignation d'un candidat plutôt « radical» qui a peiné à rassembler dans le «vrai » scrutin au-delà du noyau des sympathisants. À droite, elles ont produit, à la faveur d'une forte mobilisation (4 millions d'électeurs), un candidat un temps incontesté mais, une fois ce dernier disqualifié par le «Penelope Gate», elles l'ont protégé et empêché le recours à un candidat de substitution. Le PS a, quant à lui, dans un contexte d'anticipation de la défaite, organisé des primaires qui se sont apparentées à un pré-congrès et ont donné lieu à un vote identitaire ne prenant en compte que faiblement l'éligibilité du candidat. Elles ont désigné un candidat «frondeur» en rupture avec la ligne majoritaire de son parti qui n'a pas su ni pu fédérer l'électorat habituel du Parti socialiste. Manuel Valls a transgressé le principe même de la procédure en appelant à voter pour Emmanuel Macron. Ces deux primaires ont été marquées par un phénomène d' "entrisme» de non-sympathisants (de gauche lors de la primaire de droite, de la gauche radicale lors de la primaire de la Belle alliance populaire). La base électorale des primaires, aléatoire, rend l'issue du scrutin imprévisible. Le processus a donc largement échappé à ses organisateurs. Les primaires étaient conçues comme un outil pour produire un leadership incontesté et re-légitimer les partis en forçant l'unité. Elles ont plutôt affaibli et abîmé le candidat désigné et

25. Voir Les primaires ouvertes en France. Adoption, codification, mobilisation, R. Lefebvre, É. Treille (dir.), Rennes, Presses universitaires de Rennes, 2016.

26. Voir Les partis politiques à l'épreuve des procédures délibératives, R. Lefebvre, A. Roger (dir.), Rennes, Presses universitaires de Rennes, 2009.

27. Le point, 12 avril 2017. 
n'ont pas enclenché des dynamiques de rassemblement (comme en 2012) ${ }^{28}$.

Plus généralement, les primaires tendent à transformer les partis et à accuser certaines évolutions décrites précédemment. Elles affaiblissent encore un peu plus la fonction idéologique des partis. Non seulement le pouvoir de désignation du candidat leur échappe mais ils perdent également ce qui restait de leur fonction programmatique (en quelque sorte privatisée: chaque candidat présente un programme). La primaire contribue aussi à dévaluer le militantisme. À quoi bon militer dans un parti si les adhérents sont dépossédés de cette gratification symbolique qu'est le pouvoir d'investiture? Le rôle des militants se réduit désormais à la (lourde) fonction d'organisation de la procédure des primaires. Le parti est donc un peu plus évidé de ses fonctions traditionnelles. Au final, les primaires constituent un aveu d'impuissance des partis. Incapables de se rénover et de construire des relations avec la société sur des bases nouvelles, ils ont recours à une procédure qui non seulement les met potentiellement en danger mais compromet encore un peu plus leur légitimité dans le système politique ${ }^{29}$.

\section{Les partis-mouvements: un modèle stabilisé?}

Les partis-mouvements ou plateformes apparus à la faveur de la double consultation électorale de 2017 contestent la légitimité démocratique des primaires ${ }^{30}$. La République en marche et La France insoumise, s'ils constituent juridiquement des partis classiques pour pouvoir notamment bénéficier du financement public, cherchent plus généralement à subvertir le modèle partisan traditionnel jugé «bureaucratique» et inefficace pour régénérer une vie politique «fossilisée» par «les partis du système». Alors même qu'ils occupent des espaces idéologiques opposés, les deux mouvements présentent de nombreux points communs sur le plan organisationnel. Ils cherchent à concilier horizontalité participative et efficacité décisionnelle loin des formes traditionnelles de la démocratie partisane. La prudence doit être de mise dans l'analyse tant ces partis sont récents et leur fonctionnement encore peu stabilisé (on pourrait aussi mettre en avant leurs traits de partis présidentiels ou présidentialisés). Il s'agit ici avant tout d'observer un modèle émergent et alternatif aux partis dominants, prenant le PS comme figure repoussoir.

\section{A. Le parti-plateforme}

Les circonstances et les conditions de leur création sont proches. Les deux organisations ont été créées par le haut au moment de l'élection présidentielle par un candidat autoproclamé mais qui cherche à appuyer son ambition sur une structure collective (cette création par le haut amène d'ailleurs à nuancer le label de parti-mouvement, bottom up - «de bas en haut»...). Marquées par une très forte personnalisation, ces machines électorales ont été créés ad hoc (et ex nihilo pour En marche) pour élire un leader et donc traduisent une forme de conformation à la $\mathrm{V}^{\mathrm{e}}$ République et la place centrale de l'élection présidentielle (pour mieux la mettre en cause pour Jean-Luc Mélenchon). Pierre Rosanvallon analyse cette dimension personnelle et la rupture historique qu'elle introduit: «C'est une personne qui propose à la société de s'identifier à elle. L'offre politique détermine la demande alors qu'avant la demande était censée gouverner l'offre $»^{31}$. Emmanuel Macron l'analyse lui-même: «[...] je suis très lucide sur le fait que ce sont les Français et eux seuls qui m'ont fait et non un parti politique ${ }^{32}$. Cette personnalisation exclut tout débat ou controverse sur le leadership qui apparaît naturel. Emmanuel Macron a créé un parti qui porte ses initiales et qui est voué à son soutien avant et après l'élection ${ }^{33}$. Le leader joue un rôle essentiel et coagulateur dans la théorie du "populisme de gauche ${ }^{34}$ dont s'inspire Jean-Luc Mélenchon qui se présente volontiers comme «la clé de voûte» de La France insoumise.

Les deux mouvements consacrent une nouvelle forme de militantisme qui passe par une adhésion formelle minimale sur Internet et correspond au modèle de l'engagement distancié (intermittent, à faible coût, peu inclusif...). Les rites de passage traditionnels qui constituent les coûts d'entrées dans les partis politiques (logiques de parrainage et filtrage social) sont pensés comme des obstacles à l'adhésion et sont supprimés. L'inscription se fait sur Internet dans l'immédiateté (grâce à quelques clics) et gratuitement. La cotisation qui attestait de l'engagement

28. Voir R. Lefebvre, É. Treille, «Le déclenchement des primaires ouvertes chez Les Républicains et au Parti socialiste. Entre poids du précédent de 2011 et bricolages organisationnels (2016-2017)", Revue française de science politique, vol. 67, n 6, 2017. Benoît Hamon ne rassemble au final au premier tour de l'élection présidentielle que 25000 voix de plus que la participation à la primaire.

29. Voir R. Lefebvre, Les primaires socialistes. La fin du parti militant, Paris, Raisons d'agir, 2011.

30. Emmanuel Macron déclare: «[...] de prime abord, on se dit, la primaire c'est génial, c'est démocratique... En fait, cela crée de la tauromachie plus tôt. On fait tourner les vachettes avant d'aller à la corrida. Certains y vont pour gagner, d'autres pour perdre proprement avant d'aller à la corrida. La primaire c'est la réponse d'un système partidaire fatigué pour survivre au risque qu'est devenu le FN» (Le point, $1^{\mathrm{er}}$ septembre 2016 ) Jean-Luc Mélenchon considère quant à lui que la primaire est un moyen de préserver l'hégémonie du PS en écartant ses rivaux à gauche.

31. Le Monde, 16 septembre 2017.

32. Entretien dans La nouvelle revue française, $\mathrm{n}^{\circ} 630$, mai 2018.

33. De ce point de vue, l'affaiblissement du parlementarisme nourrit bien l'évidement des partis qui se présidentialisent. Les députés En marche sont d'autant plus dociles qu'ils doivent largement leur élection au président de la République. Les partis, comme le note Pierre Rosanvallon, représentent moins la société que «le pouvoir auprès de la société» (Le Monde, 3 mars 2017).

34. Voir E. Laclau, C. Mouffe, Hégémonie et stratégie socialiste: vers une politique démocratique radicale, Besançon, Les solitaires intempestifs, 2009. L’inspiration vient aussi ici des partis d'Amérique latine. Hugo Chávez au Venezuela se définissait comme «un homme-peuple». 
matériel du militant n'est plus obligatoire (subsiste la possibilité de faire des dons). Cette souplesse permet un « engagement » rapide et d'afficher la façade médiatique d'un parti bénéficiant d'un large soutien populaire dans une logique de monstration démocratique. En mai 2017 , La France insoumise revendique ainsi 539000 adhérents ayant laissé leurs coordonnées sur la plateforme numérique; en juillet 2017, le mouvement d'Emmanuel Macron déclare quant à lui 373 ooo marcheurs (soit bien plus que les partis en place). Résultat d'un «enclicage» simple et immédiat, l'adhésion se fluidifie, s'assouplit mais se fragilise aussi et devient volatile. Le vote sur les statuts d'En marche en juillet 2017 n'a mobilisé que 72000 votants (sur 270000 adhérents: $90 \%$ de «oui»). Un animateur de comité local d'En marche déclare à Libération, le 6 octobre 2017: «Les adhésions, ça ne veut rien dire. Vous pouvez vous inscrire au parti mais pas vous désinscrire, sauf à envoyer une lettre recommandée, ce que personne ne fait». La plateforme permet un engagement à la carte d'intensité variable qui correspond au modèle du multi-speed membership mis en évidence par Susan Scarrow ${ }^{35}$.

Internet joue un rôle essentiel dans l'économie des pratiques et du fonctionnement partisan. La plateforme numérique constitue le principal support d'une communauté partisane dans une large mesure déterritorialisée (on n'adhère pas localement comme au PS où la section communale constitue la porte d'entrée) ${ }^{36}$. La plateforme, substitut fonctionnel à la «bureaucratie » partisane (même si elle est bien pilotée, animée, encadrée...), est censée créer les conditions de l'horizontalité du mouvement en favorisant la communication de ses membres et les initiatives et un engagement à la carte et flexible dans une "philosophie de l'action et du mouvement ${ }^{37}$. Sur son blog, le 28 mai 2017, Jean-Luc Mélenchon écrit:

[...] les connexions rendues possibles par une telle plateforme vont dans tous les sens; elles sont par définition polymorphes. C'est-à-dire qu'elles ne sont ni exclusivement du haut vers le bas ni du bas vers le haut mais dans tous les sens de sorte que la notion même de haut et de bas, comme dans l'univers matériel... dépend de la position de l'observateur. [...] Tout cela, désormais, est accompli par la plate-forme sans produire aucune sorte de ces bureaucraties intermédiaires hier encore indispensables à la marche ordinaire de la vie quotidienne ${ }^{38}$.
Les deux mouvements s'appuient par ailleurs sur une structure souple qui cherche à combiner horizontalité et centralisation décisionnelle, engagement sur le terrain et efficacité. Il s'agit d'éviter par des règles prophylactiques «la bureaucratisation», pente naturelle des partis. Deux écueils sont à conjurer: la constitution de baronnies locales et la formation de sensibilités internes qui ankylosent l'organisation. Le PS dont sont issus de nombreux dirigeants des deux organisations ${ }^{39}$ est ici érigé en contremodèle. L'autonomisation de ses notables combinée à sa structuration en courants aurait bloqué le parti. S'il s'appuie sur un référentiel négatif, le modèle organisationnel apparaît pourtant dans les deux cas encore mal défini et flottant, surtout pour La France insoumise. Michel Offerlé évoque à propos d'En marche «une structure agrégative non encore stabilisée, entre ferveur et spontanéité militante des premiers temps, opacité organisationnelle, horizontalité dans une bonne volonté numérique et verticalité revendiquée $»^{40}$. La France insoumise est toujours un work in progress qui développe une réflexion en actes et in situ sur la forme partisane stimulée par une imagination organisationnelle qui n'exclut pas tâtonnements et bricolages ${ }^{41}$.

La République en marche se dote de statuts en juillet 2017. Le conseil national, parlement non élu du parti, est composé à $80 \%$ des parlementaires, référents territoriaux et représentants des territoires (membres de droit) et à $25 \%$ de conseillers tirés au sort «à intervalles réguliers" parmi les adhérents. Le conseil élit en son sein la direction nationale: vingt des membres du bureau exécutif et le(s) délégué(s) général(ux). Dix membres du bureau exécutif sont cooptés par ses membres. La direction nationale nomme les référents territoriaux (représentants du mouvement au niveau départemental) et la commission d'investiture des candidats aux élections. Une très large majorité des membres du conseil national qui élit la direction nationale procède donc de la direction nationale. Notons aussi que la légitimité des instances nationales et locales ne découle aucunement de leur élection directe ou indirecte par les adhérents. Le délégué général de La République en marche, Christophe Castaner, a été désigné à mains levées en novembre 2017. Une des dirigeantes, Catherine Barbaroux, justifie ces choix par une volonté de limiter «les ambitions pas forcément tournées vers l'intérêt du mouvement ${ }^{42}$. L'avocat JeanPierre Mignard, proche de François Hollande et longtemps adhérent du PS, qui a participé à la rédaction des statuts

35. S. Scarrow, Beyond Party Members: Changing Approaches to Partisan Mobilization, Oxford, Oxford University Press, 2015.

36. «Le communisme disait Lénine, c'est les soviets plus l'électricité, les mouvementistes ce sont un chef plus internet», note le politiste Michel Offerlé («Les partis meurent longtemps», Le Monde, 31 mai 2017).

37. Jean-Luc Mélenchon convient que la structuration des deux mouvements est proche mais que «le rapport des deux plateformes avec la société n'est pas le même» (blog du 4 mars 2017, https://melenchon.fr/2017/o3/o4/des-nouvelles-du-mouvement).

38. Jean-Luc Mélenchon, blog du 28 mai 2017, https://melenchon.fr/2017/05/28/a-propos-du-mouvement-la-france-insoumise.

39. La socialisation partisane du délégué général de La République en marche, Christophe Castaner, comme celle de Jean-Luc Mélenchon et de son entourage, ont été marquées par leur engagement au PS.

40. M. Offerlé, «Les partis meurent longtemps».

41. Voir, notamment, «La France Insoumise doit se glisser dans tous les interstices de la société - Entretien avec Manuel Bompard», Le vent se lève, 21 septembre 2017.

42. Marianne, 10 juillet 2017. Les adhérents sont néanmoins directement consultés par le bureau exécutif sur les sujets de son choix et ceux qui seront proposés par $20 \%$ des adhérents ou comités locaux. Les adhérents peuvent également sélectionner les actions financées par le budget participatif du mouvement. 
déclare que les référents locaux ne peuvent pas avoir de mandat électif "pour éviter que ne se créent des baronnies locales » ${ }^{43}$. Le même assume la «centralisation» du parti qui «doit permettre d'éviter une pétrification du débat d'idées dans des représentations de courants, comme on a pu l'observer au PS ou chez LR». Il ajoute: «Notre souci a été d'assurer la cohérence entre la ligne du parti et le Président ${ }^{44}$.

La France insoumise ne dispose pas officiellement de statuts $^{45}$. Aucune direction n'est identifiable (le groupe parlementaire présidé par Jean-Luc Mélenchon en tient lieu). L'équipe opérationnelle dirigée par Manuel Bompard ne procède d'aucune élection. La structure de base est le groupe d'appui mais l'ensemble du mouvement apparaît peu hiérarchisé. Il s'agit là encore d'échapper à la lourdeur bureaucratique, à l'empilage des structures intermédiaires par une organisation en rhizome, faisant place à l'autonomie, à la réactivité, à l'esprit d'initiative. Le député Adrien Quatennens commente cette situation:

Notre force, c'est que les outils qu'on continue à développer empêchent que s'installe une bureaucratie locale. Pendant qu'on se regarde le nombril, on laisse de l'espace à l'extérieur. Il faut qu'on continue à s'organiser par l'action, et qu'on soit projetés sur l'extérieur. [...] Plus notre mouvement restera élastique, souple, malléable, plus on sera fort. Plus on cherchera à se structurer, plus on sera fragile ${ }^{46}$.

Jean-Luc Mélenchon définit en ces termes La France insoumise dans un entretien très riche consacré à sa conception du mouvement:

C'est un mouvement. Nous ne voulons pas être un parti. Le parti, c'est l'outil de classe. Le mouvement est la forme organisée du peuple. L'idée, c'est d'articuler le mouvement, sa forme et son expression: le réseau [...]. Le but du mouvement de la France insoumise n'est pas d'être démocratique mais collectif. Il refuse d'être clivant, il veut être inclusif. [...] Le mouvement n'est ni vertical ni horizontal, il est gazeux. C'est-à-dire que les points se connectent de façon transversale: on peut avoir un bout de sommet, un bout de base, un bout de base qui devient un sommet ${ }^{47}$.

Les formes habituelles de "démocratie partisane» interne sont mises à distance dans les deux mouvements. L'absence d'élections internes, autres que des consultations thématiques, est théorisée. Les investitures aux élections législatives ont été centralisées et n'ont pas donné lieu à vote des adhérents. Elles ont été supervisées à En marche par une commission nationale, présidée par Jean-Paul Delevoye, qui a statué de manière discrétionnaire sur la base des curriculum vitae des candidats à la candidature et d'entretiens de recrutement sur un mode managérial, principalement par téléphone. Les comités d'En marche peuvent se créer sans contrainte mais leurs référents sont nommés. Aucun vote interne n'est prévu pour désigner les dirigeants. Le rôle des adhérents selon Arnaud Leroy, membre de la direction collégiale, est d' « expliquer l'action de l'exécutif et de remonter les attentes et ressentis des Français ${ }^{48}$. Le tirage au sort, forme de concession démocratique, est dans les deux cas censé à la fois assurer la présence d'adhérents de base (profanes) dans les instances et conjurer le risque de l'oligarchisation de l'organisation. Des insoumis sont tirés au sort pour participer à des conventions thématiques. Les règles des deux organisations ne font aucunement droit à un pluralisme organisé.

D'après Jean-Luc Mélenchon, dans un entretien à l'hebdomadaire Le 1, «le programme est le socle» ${ }^{49}$. Il écrit dans son blog du 28 mai 2017:

[...] les processus de «démocratie interne» sont également à l'œuvre. Mais dans le mouvement, on s'efforce de ne jamais en faire un sujet de conflictualité interne. Il n'y a donc pas de "majorité», de "minorités», pas de plateformes concurrentes, pas d'orientation générale opposée les unes aux autres. Autrement dit: le mouvement se soucie d'abord d'être inclusif et collectif davantage que formellement "démocratique», sachant à quelles violences et dérives conduisent les soi-disant pratiques «démocratiques » organisées par les règlements intérieurs des partis traditionnels ${ }^{50}$.

\section{B. Des cultures militantes opposées}

Les deux mouvements se différencient néanmoins du point de vue des cultures militantes (on ne traite pas ici de leurs options idéologiques très opposées).

La culture managériale est centrale à La République en marche qui fait sien le modèle de la startup numérique, «souple» et «agile», constitutif d'une nouvelle forme de «parti-entreprise» relativement inédite en France ${ }^{51}$. La difficulté est ici de saisir les pratiques partisanes concrètement au-delà d'une rhétorique managériale et de la revendication légitimante d' « efficience» et de modernité. Le discours entrepreneurial est en tout cas omniprésent chez En marche pendant la campagne présidentielle ${ }^{52}$ ( briefs », «notes de synthèse », «bonnes pratiques»...). Les volontaires sont alors structurés en «teams». Les «campaigners» "mappent» (planifient), «targetent»,

43. Le Monde, 8 juillet 2017.

44. Libération, $\mathbf{1}^{\mathrm{er}}$ août 2017

45. La première convention nationale après les élections présidentielle et législatives du mouvement La France insoumise à Clermont-Ferrand les 25 et 26 novembre 2017 n'a pas vraiment apporté de réponses.

46. Le Parisien, 27 août 2017

47. Le 1, octobre 2017 .

48. Le Monde, 7 septembre 2017

49. Le 1, octobre 2017.

50. Jean-Luc Mélenchon, blog du 28 mai 2017, https://melenchon.fr/2017/05/28/a-propos-du-mouvement-la-france-insoumise.

51. Voir J. Hopkin, C. Paolucci, "The Business Firm Model of Party Organisation: Cases From Spain and Italy", European Journal of Political Research, vol. 35, n 3, 1999, p. 307-339.

52. Voir M. Magnaudeix, Macron \& Cie: enquête sur le nouveau président de la République, Paris, Don Quichotte, 2017. 
«benchmarkent »... En marche est donné à voir comme une organisation «from scratch» (lancée à partir de rien), une structure qui n'est pas «top down» (verticale) mais bien «bottom up» ( "de bas en haut»). Dans les meetings, les «helpers" doivent veiller à la "Team Ambiance». Parmi les adhérents, un vivier de 12 ooo personnes, volontaires pour s'impliquer davantage, est suivi par une «cellule $\mathrm{RH}$ ». Lors de la campagne présidentielle, le siège (40 permanents sont recrutés rapidement) est dominé à sa tête par de nombreux cadres issus des écoles de commerce formés à la gestion des entreprises et des ressources humaines. Le plus proche entourage du candidat est issu des plus grandes écoles de commerce plus que de l'École nationale d'administration. Emmanuel Macron use méthodiquement des usages récents des open data, des outils de géolocalisation et des logiciels à algorithme. Les comités locaux organisent des «challenges» (défis). Le mouvement est censé répondre avec application aux mots d'ordre du toyotisme fondé sur le «zéro défaut, zéro temps mort, zéro stock». Pendant la campagne, la centaine de référents départementaux ont été recrutés sur curriculum vitae, par le pôle «territoires » du mouvement, avant d'être confirmés par Emmanuel Macron et le secrétaire général du mouvement, le député socialiste Richard Ferrand. Emmanuel Macron confie à Mediapart le 13 janvier 2017:

Une des choses qui affaiblit les partis politiques actuels, c'est le manque de professionnalisme de leurs réponses à leurs adhérents. Les partis sont là pour transformer le réel mais aussi donner une place à chacun, pour animer les gens. Quand ils ne le font pas, c'est très décevant. Donc il faut être très professionnel et très organisé, j'y attache beaucoup d'importance.

Après l'élection présidentielle, des rapports d'activité individualisés sont envoyés aux adhérents d'En marche. Dans le fonctionnement interne, les «compétences" des marcheurs qu'ils font valoir sur le site Internet sont valorisées pour accroître l'efficacité de l'organisation. On observe néanmoins une démobilisation très forte des marcheurs après l'élection présidentielle, perceptible notamment au moment de l'opération «La marche pour l'Europe» en avril 2018. La République en marche n'estelle avant tout qu'un club de mobilisation électorale?

La France insoumise valorise quant à elle un activisme militant à la fois idéologique et pragmatique. Il s'agit de mettre en mouvement en permanence les forces militantes et de nourrir une dynamique constamment entretenue («l'efficacité», tournée vers l'action, est aussi revendiquée) ${ }^{53}$. La conception de l'engagement insoumis est traversée de tensions entre une forme d'avant-gardisme que Jean-Luc Mélenchon a incorporée dans ses expériences partisanes précédentes (la «conscientisation du peuple» par des instances extérieures à lui) et le spontanéisme et la démocratie participative (la marque de fabrique du mouvement est selon lui «l'auto-organisation populaire»). Le mouvement est censé sortir aussi d'une conception purement électoraliste de l'activité partisane (être un mouvement «supra-électoral»), qui est celle des partis traditionnels. L'organisation s'appuie pour ce faire sur des répertoires militants anciens (issus des organisations du mouvement ouvrier ${ }^{54}$ ) mais aussi d'autres hybrides ou inédits. La dimension idéologique de l'engagement est affirmée: elle conduit à mettre en avant «la bataille culturelle», «la conquête des esprits», la diffusion d'un contre-imaginaire au libéralisme (promotion d'une contreculture, utilisation de contre-media...) par des actions de formation notamment ${ }^{55}$. Mais le militantisme doit aussi se matérialiser dans des combats concrets et prendre sa place dans le quotidien des groupes vulnérables cibles par des actions de service et de solidarité immédiate (soutien scolaire, distributions...). À l'été 2017, les «caravanes des droits » sillonnent les quartiers populaires pour informer les habitants sur leurs droits et leur permettre de réaliser des simulations afin d'accéder à l'intégralité de leurs droits sociaux. Elles doivent permettre aussi de mobiliser contre la réforme du Code du travail. Jean-Luc Mélenchon s'en explique dans l'hebdomadaire $L e 1$ :

On a repris l'idée de caravanes militantes, qui vont à la rencontre des gens, qui les renseignent sur les droits sociaux dont ils peuvent bénéficier, qui les inscrivent sur les listes électorales, qui amènent des écrivains publics et des équipes sanitaires, qui mènent la bataille contre les punaises de lit ! On ne peut plus aujourd'hui se contenter de mettre une pile de prospectus sur une table et d'attendre le chaland. Il faut créer les équipes de potes, aller faire du porte-à-porte, et surtout rester et discuter ${ }^{56}$.

Les groupes d'appui doivent s'ancrer dans les formes de conflictualité locale (luttes contre les bailleurs sociaux, les fermetures d'usines ou de classes). Un nouveau folklore militant émerge, autour des «casserolades» par exemple, analysées par Jean-Luc Mélenchon comme de nouveaux « rites d'identification » militante. L'importation de formes de community organizing doit permettre d'organiser les doléances, de former des contre-pouvoirs

53. Cet activisme doit permettre de marginaliser les rivaux à gauche de Jean-Luc Mélenchon. La France insoumise, au-delà de sa modernité affichée, est ainsi "plus prosaïquement une entreprise de captation et de réorganisation de l'espace politique à gauche» (M. Offerlé, «Les partis meurent longtemps»). 54. Jean-Luc Mélenchon réactive ainsi le concept de "contre-société» attachée historiquement à la forme partisane communiste.

55. Voir R. Lefebvre, Après la défaite. Analyse critique de la rénovation au Parti socialiste, Paris, Fondation Jean Jaurès, 2018. Olivier Faure, nouveau premier secrétaire socialiste, a proposé de lancer une plateforme numérique au PS et de revoir de fonds en combles son modèle. Les réponses organisationnelles des partis à leurs crises de légitimité empruntent deux trajectoires qui apparaissent $a$ priori contradictoires: d'un côté, elles s'inscrivent dans un processus de professionnalisation accrue qui présuppose le recours à des professionnels (de la communication et du marketing) et au numérique. De l'autre, elles passent par la mise en place de procédures visant au contraire à contourner les structures intermédiaires entre l'«élite» et le «peuple» et à promouvoir la démocratie participative sous des formes diverses. Voir A. Gauja, Party Reform. The Causes, Challenges, and Consequences of Organizational Change, Oxford, Oxford University Press, 2017. Voir aussi les travaux en cours d'Agnès Alexandre-Collier.

56. Le 1, octobre 2017 
par le porte-à-porte notamment. Mais des campagnes nationales doivent aussi faire croître le mouvement à une échelle plus globale, notamment à travers l'organisation de votations citoyennes (31500 participants se prononcent à 93,13\% de « oui » sur la sortie du nucléaire en mars 2018 dans 2000 lieux physiques ou sur Internet).

\section{Conclusion}

Les partis sont confrontés, en France comme en Europe, à une crise de légitimité sans précédent, même si leur légitimité a toujours été discutée au cours de leur histoire. Les configurations classiques du jeu partisan sont déstabilisées par l'émergence de nouvelles forces politiques, à gauche comme à droite, qui contestent le «système» et les élites à partir de positions extérieures à eux mais aussi à partir du système lui-même ${ }^{57}$. Encastrés dans la démocratie représentative dont ils sont un des médiateurs, les partis sont emportés par sa mise en cause et sa fragilisation. Pépinières de professionnels de la politique, ils sont stigmatisés comme des structures élitistes d'autant plus que leur ancrage dans la société, à travers le militantisme notamment, s'est fortement affaissé. Les partis ne parviennent plus à organiser la vie politique à partir de la vie sociale, à donner une expression politique à une société qu'ils ne représentent plus ou ne parviennent plus à mettre en forme.

Face à ce discrédit, les partis ne restent pas inactifs. Issus souvent de longues traditions historiques, les partis établis, de plus en plus affaiblis, cherchent à se réinventer, se rénover, se refonder ${ }^{58} \ldots$ en s'ouvrant sur la société ou leurs « sympathisants» au risque de mobiliser de nouveaux instruments potentiellement destructeurs (les primaires). Avec la transformation des modes de communication, la forme "parti politique», hiérarchisée et disciplinée («caporalisée»), apparaît frappée d'obsolescence mais elle perdure. L'heure est au réticulaire, à l'agilité, à l'efficience, à la réactivité, à l'interactivité, à l'individualité pour dessiner le parti «post-moderne » et «uberisé ». Les partis-mouvements apparaissent plus plastiques, adaptables et en prise avec «les demandes» de la société. Ils se construisent en rupture avec le modèle démocratique partisan classique et le "vieux monde " partisan encalminé au risque de générer de nouvelles formes de dépossession, d'élitisation et de personnalisation ${ }^{59}$. Tout en s'appuyant sur les règles institutionnelles existantes qui leur permettent de rafler la mise, ils combinent individualisation de l'offre de militantisme et extrême centralisation et personnalisation du leadership. Résisteront-ils à la loi d'airain de la normalisation qui guette toute nouvelle organisation $^{60}$ ? Les élections municipales seront sans doute décisives pour l'évolution de La France insoumise et En marche. Comment ces mouvements vont-ils faire face à la nécessité de produire des élites locales, de s'implanter au risque de la notabilité et d'encadrer la professionnalisation politique qui peut en découler? Comment par ailleurs vont-ils réguler les divergences internes qui peuvent apparaître avec le temps? Les tentatives de dépassement de la forme partisane se multiplient. Il faut faire parti, mais autrement, en s'inspirant d'expériences étrangères (Podemos, le Mouvement 5 étoiles...).

Mais peut-on, plus généralement, imaginer un au-delà du parti ? Les partis politiques sont à la fois dévitalisés et incrustés dans le jeu politique, désencastrés socialement et enkystés institutionnellement. Sans l'essentialiser, la forme partisane paraît consubstantielle à la démocratie électorale et, tant que les systèmes politiques seront centrés sur la procédure électorale, on imagine mal comment ils pourraient en faire l'économie (nécessité de coordonner des acteurs et des élus, de produire des choix, de structurer l'offre politique, de collecter des moyens...). Il ne faut sans doute plus penser les partis politiques selon le modèle partisan, historiquement situé, des partis de gauche de la fin du XIX ${ }^{\mathrm{e}}$ siècle et interroger à nouveaux frais les cadres analytiques de la sociologie des partis. Les partis ne sont qu'une des formes historiquement déterminées d'entreprise politique et les formes de collectivisation des ressources politiques sont appelées à évoluer.

57. Emmanuel Macron apparaît comme un «maverick» pour reprendre l'expression du politiste Robert R. Barr qui désigne par là un franc-tireur, "membre de la structure de pouvoir établie qui rompt avec elle de l'intérieur " (R. R. Barr, "Populists, Outsiders and Anti-Establishment Politics », Party Politics, vol. 15, nº 1, 2009, p. 34; nous traduisons). Il subvertit le cursus honorum traditionnel tout en provenant de la noblesse d'État. Emmanuel Macron déclare à Challenges, le 16 octobre 2016: «Le système politique, avec ses codes et ses usages, je ne cherche pas à le respecter parce que je ne lui appartiens pas. Ça ne me pose aucun problème de transgresser ses codes. Ma volonté de transgression est d'autant plus forte que j'ai vu le système de l'intérieur».

58. Des écoles du mouvement sont lancées en février 2018, coordonnées par le politiste Thomas Guénolé qui convoque la tradition de l'«éducation populaire».

59. Voir J. Fretel, «L'avenir des illusions partisanes», Savoir/ agir, $\mathrm{n}^{\circ} 32$, juin 2015, Réinventer les partis politiques, N. Éthuin, R. Lefebvre (dir.), p. 45-52.

60. «[...] entreprises de représentation nécessaires au fonctionnement de la démocratie», les partis se transforment facilement en «entreprises de dépossession des intérêts de ceux qui placent en eux leur confiance» (Y. Poirmeur, Les partis politiques: du XIX ${ }^{e}$ au XXI ${ }^{e}$ siècle en France, Issy-les-Moulineaux, LGDJ, 2014, p. 168-169). 